\title{
Capacity Management for Internet Traffic
}

\author{
S. O. Larsson and A. A. Nilsson \\ Dept. of Telecommunications and Signal Processing \\ University of Karlskrona/Ronneby \\ Sweden
}

\begin{abstract}
We describe methods to guarantee a certain level of service for Internet traffic by reserving capacity along fixed logical paths. The amount of needed capacity is calculated by using a trade-off between connection rejection probability and the utilization of the capacity. This can be used in the process of dimensioning capacity on a long term basis. Two dynamic allocation methods are proposed, which periodically reallocates capacity according to measured traffic loads. Call admission control is used and automatic connection retrials are studied. Each method is designed for a particular scenario. In the first one, a LAN reserves capacity to get a certain transmission speed for every connection. Capacity is paid for according to how much of it that is reserved. The second one considers an aggregated traffic generated by users having limited transmission speed in their connection to the Internet (e.g. modem- and mobileusers) and the operator of the network manages the capacity. The Internet traffic is modeled as Web page fetches from the World Wide Web.
\end{abstract}

Keywords: Internet Traffic, QOS, Capacity Dimensioning, Resource Allocation.

\section{Introduction}

As the global Internet grows so does the demand for capacity. There is also a desire to incorporate real-time services and thereby extending the best effort class of service to multiple classes of services. For near real-time applications (e.g. IP Telephony) some level of quality of service (QoS) is needed. Reservation of capacity can accomplish this. To be able to guarantee a maximum delay variation and maximum packet loss probability one should not setup more connections than can be handled. A connection admission control (CAC) algorithm controls that the QoS will be met and subsequently rejecting connection at some times when the load is high. The reservation is best done along a fixed path. Internet has traditionally only supported a single best-effort traffic. It is based on a connection-less packet-networking infrastructure. Packets can always be sent but no guarantees are given that they will arrive to the destination. This depends on the stateless Internet protocol (IP) infrastructure and an inadequate support from network controls. By building a statebased infrastructure and using a connection-oriented approach, support can be given for QoS guarantees.

Lots of effort has been put into developing protocols to support this. ATM Forum has developed the Multiprotocol over ATM (MPOA), while IETF has developed the Multiprotocol label switching (MPLS). MPLS enables labelled switched paths (LSPs) over Internet. Compared to virtual path connections in an ATM network, the LSPs 
can be used "inside" other LSPs, i.e. used in hierarchical levels or layers [12]. The logical paths also enables fast switching of the data packets since the routing already has been done. With new services for mobile users with limited capacity, reservation of capacity will give the users value for their money. The amount of reserved capacity should also be adjusted according to actual traffic load. The reservations can be achieved with the proposed integrated services (IntServ) framework [13]. It will keep track of the flow-state (with additional regular updates) for each connection by the use of the resource reservation protocol (RSVP) [14]. At the edges of a core network, individual buffers ensure that connections are protected against each other, but the resources should be reserved for an aggregate of users in the core network. Considering the scaling problem with IntServ, IETF has proposed a different framework called differentiated services (DiffServ) [16]. In this case, packets are tagged with priority according to delay requirements and by value of importance. The tag is used by core routers for scheduling, handling congestion, and so on, on a hopby-hop basis. This will work as long as enough capacity is available. To decrease the packet loss for low priority packets in high load situations or when other traffics misbehave, some sort of intelligent scheduling mechanism must be used. Weighted fair queuing (WFQ) scheduling has been proposed for these purposes. This management system supports several classes of services between best-effort and guaranteed services. IETF's controlled-load services [15] is a guideline about how to support real-time applications with a degree of service that corresponds to a moderate loaded network. A CAC algorithm is also introduced. To be able to guarantee QoS, our proposed method for capacity reservation will be useful for ensuring that not more traffic, than can be handled, is injected into the network.

We assume that users have limited bandwidth for the transmission of data (e.g. by the modem speed) and that LSPs (or similar) for which capacity are reserved, are used for these users. This means that we use constant transmission speeds. It has been assumed that the transmission speed is controlled by some mechanism on the sending side [6]. For IP Telephony this is obvious but it can be used for the most data transfers and with this approach we do not suffer from slow start, round-trip delay and other problems as compared to TCP. Capacity sharing and utilization accomplished with TCP is an important aspect that should be used for some services. In the following, a lower limit for the transmission speed can be used instead of a constant transmission speed.

Section 2 describes our model and assumptions of the traffic. It is difficult to dimension networks to cope with Internet traffic, since this traffic is fundamentally different from traditional voice traffic. Over-provisioning of capacities is one way to postpone the problem. In Section 3 a strategy for the dimensioning problem is proposed. Even if a proper design can be found for steady traffic, the ever-changing traffic should be handled efficiently. If capacity is reallocated dynamically the network will be better utilized. In Section 4 we propose an allocation function for Web traffic destined to a certain LAN, while in Section 5 we propose a management of the capacity needed for an aggregated stream of Web traffic to be routed through the Internet. 


\section{Traffic Model}

The traffic model used is a model of how Web pages are fetched through a Web browser. Characterization of Web traffic involves both the structure and size of the pages. Each page is designed around a main "anchor" page and a possible additional set of embedded items that appear within this anchor page. The embedded items are fetched immediately after the anchor page and are shown together with it, on the user screen.

The Web page contents are classified into two groups: HTML and non-HTML. This is only natural because of the nature and intent of the participating items. The embedded items are mostly non-text in nature and are glued together using HTML text. The modelling process of the file sizes, corresponding to the two classes, also brings out their inherent characteristic diff-erences. The non-HTML type is more variable than the HTML type. Examples of embedded items can be either: Java scripts, frames, maps etc. for HTML and Java applets, postscripts, images etc. for non-HTML. The probability of fetching HTML- and non HTML-items is set to 50\% for both.

The distributions for the file sizes and the number of embedded items have been computed in [1] from data collected by probing a broad spectrum of Web servers (e.g. newspapers, universities, search engines, commercial sites etc.). The contents from the servers were successively downloaded and analyzed for their structural information. (Only unique items were selected.) We assume that the file contents and what is actually transferred through the network is very much the same. No regards has been taken to the cache used in Web browsers.

Some of the items can be very large. The file size $(X)$ is modelled with a Pareto distribution in [2,3]. In the following, we consider the case of a combination of a uniform distribution and a truncated Pareto distribution for the file sizes, which corresponds to measurements done on commercial servers [4]. In our simulation experiments we have used the following distribution:

$$
P\{X \leq x\}= \begin{cases}\frac{(x-a)}{(b-a)} p a, & a \leq x \leq b \\ \left(\frac{1-(b l x)^{\alpha}}{1-(b l N)^{\alpha}}\right)(1-p a)+p a, & b \leq N\end{cases}
$$

According to [1] the parameters for HTML items are: $a=100, b=4200, \alpha=1.36$, and the mean file size is 12888 bytes. The probability ${ }_{p a}$ denotes the probability that $a \leq b$, and it is calculated from the total mean file size and is 0.215. For non-HTML items $a=100, b=1024, \alpha=1.06$, the mean file size is 12000 bytes, and $p a$ is almost zero.

Measurements show that the number of embedded items $Z$ in a Web page can be modelled by a negative binomial distribution [1]:

$$
P\{Z=x\}=\left(\begin{array}{c}
\delta+x-1 \\
\delta-1
\end{array}\right)\left(\frac{\beta}{1+\beta}\right)^{x}\left(1-\frac{\beta}{1+\beta}\right)^{\delta},
$$


where $\delta=1.386, \beta=6.985$. The probability to have no embedded items is set to $12 \%$ and the mean number of embedded items is ten. We assume that for a certain Web page the number of embedded items and their file sizes are independent.

The evaluation of the capacity management methods is done by simulations. Traffic is generated by Web page fetches where the embedded items are transferred in parallel after the reception of the "anchor" page. If a new connection request is rejected, the application will try again a maximum of $R_{\max }$ times. This is only natural since an upper limit of connection setup delay is wanted. $R_{\max }$ could be made to depend on the type of service. The waiting time to the next connection attempt is doubled each retrial. The delay has been set to one second for the first retrial. This means that if $R_{\max }=6$, then if a transfer of an item has not started within one minute, it will not be fetched (unless the user retry manually). The truncation of the Pareto distribution by $N$ can not be smaller than $\approx 8 \cdot 10^{10}$ if the mean value for the non-HTML file sizes is to be kept. This still means that for the simulations, a large "warm up" period is needed to measure the correct value of the mean number of connections. This is caused by the very slow addition of very long connections. As an example, assume a constant traffic intensity that suddenly changes to a new level. This will result in a long settling time to reach a new steady state, i.e. when the mean number of connections changes very slowly. By adding a couple of persistent connections at start up, the warm up period can be decreased while still having a correct mean value of the number of connections. This is particularly useful when dimensioning the capacity for steady state traffic. In the sequel, $95 \%$ confidence intervals are presented for the simulated and measured parameters.

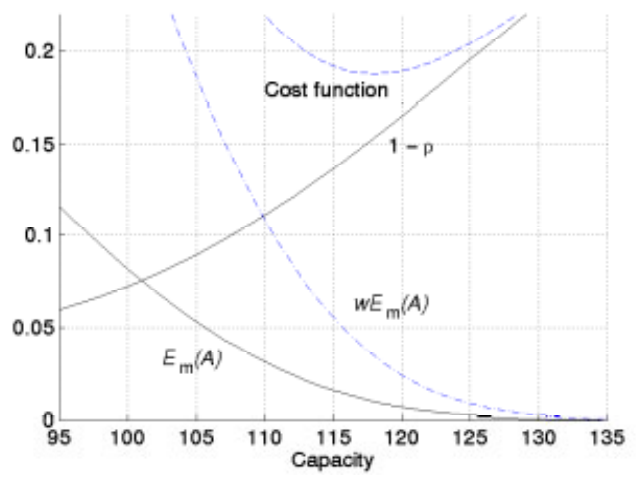

Fig. 1. Cost function and its components for voice traffic.

\section{Dimensioning}

In order to simplify the evaluation, i.e. to reduce the needed simulation time, we have not limit our investigation to a particular transport protocol. The scope is to dimension the transmission capacity of the links and the logical paths. To dimension resources, the distribution of the number of simultaneous connections has to be known. We 
denote the probability for a certain number of ongoing connections (i.e. the used capacity), as the occupation distribution. The variance of the occupation distribution when having batch arrivals is larger compared to the case with single arrivals. In order to dimension the links, we first evaluate the case with voice traffic. A trade-off is done between the utilization of the capacity and the connection setup blocking probability (CBP). By doing this we can find a relationship between the utilization and CBP, which we can apply on any type of traffic. We can also state that this will take into consideration both the network provider and the users point of views. For simplicity, we assume that the provider wants to maximize the utilization. Alternatively, let us consider that, by having this view, we get a minimum capacity at the end that can be used when dimensioning a network to meet a certain service level. Increasing the utilization will also increase the CBP. The users want a low CBP but this on the other hand will give a low utilization. It should be noted that best-effort traffic will increase the utilization further but we do not take this into account. We get the following cost function $(C)$ to minimize:

$$
C=1-\rho+w E_{m}(A),
$$

where $\rho$ is the utilization defined as:

$$
A\left(1-E_{m}(A)\right) / m \text {. }
$$

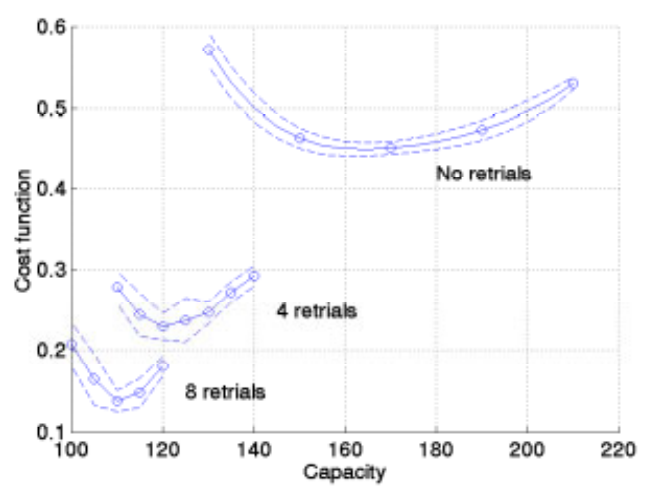

Fig. 2. Cost function for Internet traffic.

The Erlang-B formula is used, where $E_{m}(A)$ is the CBP given a traffic load of $A$ and a capacity of $m$ (maximal number of simultaneous connections). For a given $w$, the $m$ that minimizes the cost function, is denoted as the target capacity and the resulting $\mathrm{CBP}$ as the target $\mathrm{CBP}$. To obtain the value of $w$, we study the case of a voice traffic where the offered traffic is Poissonian. If we let calls be blocked with a probability of $\approx 1 \%$ for a selected traffic load of 100 , then a weighting factor $w$ can be found that optimizes (1). A weight of $\sim 3.5$ gives the results shown in Figure 1. The cost function, 1- $\rho$, and the CBP are shown. By using this weight, the needed capacity can finally be found for our model of Internet traffic. The optimum for different number of retrials has been found by simulations. Figure 2 shows the result with a 
traffic of 100. The capacity is in this case expressed as units of $56 \mathrm{~kb} / \mathrm{s}$. The dashed lines show the confidence intervals.

The capacity needed to optimize $C$ when allowing up to eight retrials is for a traffic of 100, 200, and 300: 110, 213, and 315 respectively. The target blocking probabilities are: $0.8 \%-1.1 \%, 0.3 \%-0.6 \%$, and $0.4 \%-0.9 \%$ respectively. The corresponding target capacities are shown in Figures 3 and 4. Figure 4 shows the dependence to the amount of traffic. Figure 3 shows the dependence on the number of retrials for the traffics 100,200 , and 300 . For this traffic range, using a maximum of about five retrials, the target capacities are almost equal to the capacity giving $1 \%$ blocking (calculated with Erlang-B formula) for a Poisson traffic without retrials and using a negative exponential service time of one. These capacities are shown as solid horizontal lines in Figure 3.

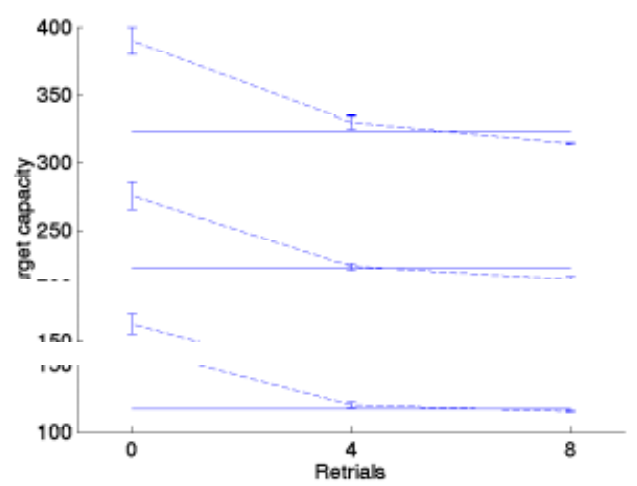

Fig. 3. Target capacity for different number of retrials.

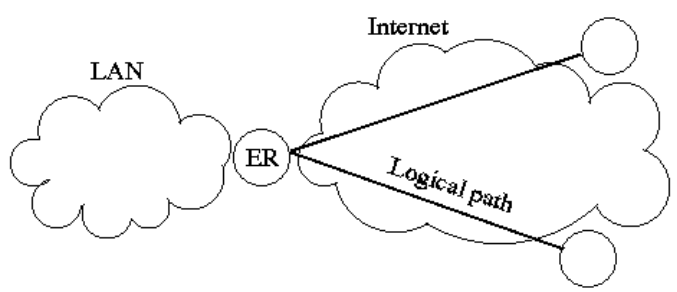

Fig. 4. Model of the network configuration.

\section{LAN - Internet}

\subsection{Allocation Function}

Figure 5 shows the case when a LAN is connected to the Internet via an edge router (ER) [7] or some sort of a label-switching router (LSR). Our goal is to calculate the needed capacity for the logical paths (e.g. LSPs) so that they can accommodate the traffic destined for the LAN. In this case, only Web browsing is considered. One can 
also see the reservation as a possible way to manage capacity for a virtual private network (VPN), where the owner of the VPN pay in relation to the capacity reserved. The ER is used for CAC, which means that connections can only be established if there is enough reserved capacity left to accommodate them. One way to introduce explicit requests that can be counted, is by letting the end-terminals send requests to the ER before connection establishment. This could correspond to the proposal in [7] for setting up IP Telephony connections by asking the ER. It can seem as a very costly solution to make connection requests and CAC for every fetch of a Web page item, however, this can and should be simplified compared to an IP Telephony connection setup. In [8] computers monitors the traffic in a campus LAN. A connection rejection is implemented by issuing a TCP RST messages during the TCP connection establishment phase. This way they can control how the capacity on the Internet access link is to be divided between the students and the staff.

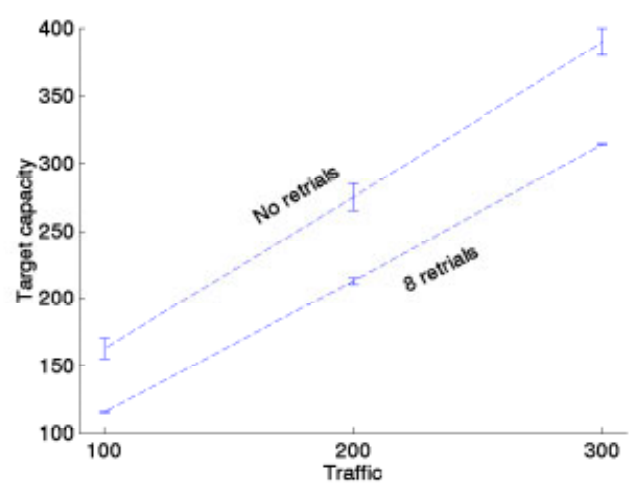

Fig. 5. Target capacity for different traffics.

The connection requests are used for estimating the traffic load, which in turn is used for the reservation. The capacity reallocations are done periodically with a period of $T_{\mathrm{u}}$ and the actual traffic load is estimated each minute.

Given the transfer rate as well as a knowledge of the mean file sizes, we can calculate the mean connection time $\bar{x}$. The mean number of simultaneous connections is $A \cdot \bar{x} \cdot 10$, since the mean number of embedded items per page is ten.

An important aspect of Web traffic is the occurrence of very long lasting connections. When reallocating the capacity periodically, these connections will have very little impact on the performance of the reallocations. Simulations have shown that in a smaller time scale and allowing for retrials, the needed capacity that minimizes the cost function is almost the same as the traffic load. The problem then is to estimate the traffic from measuring the total number of connection requests (with retrials included).

To model the connection requests we add automatic retrials when connection requests are rejected by the CAC. Figure 6 shows fresh new connection requests $(A$ or "first traffic") and the retrials $\left(A_{\mathrm{R}}\right)$. To get an estimate $\hat{A}$ of the fresh traffic $A$ we use:

$$
p=E_{m}\left(A_{\text {total }}\right)
$$




$$
A_{\text {total }}=A\left(1+p+p^{2}+\ldots+p^{R_{\max }}\right)
$$

$$
\neg=A_{\text {total }} \frac{1-p}{1-p^{R_{\max }+1}}
$$

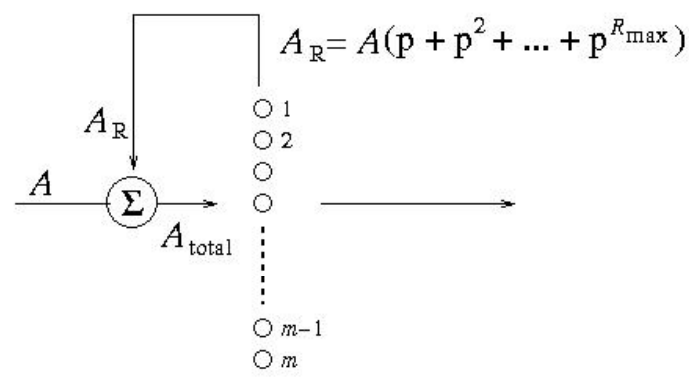

Fig. 6. Model of the retrials.

Table 1. Cost function comparisons.

\begin{tabular}{|c|c|c|c|}
\hline $\begin{array}{c}\text { Expected } \\
\text { service time } \\
\text { [secs.] }\end{array}$ & $\begin{array}{c}\text { Utilization } \\
{[\%]}\end{array}$ & CBP $\quad[\%]$ & $\begin{array}{c}\text { Cost- } \\
\text { function }\end{array}$ \\
\hline 13.5 & $92.08 \pm 1.38$ & $0.25 \pm 0.03$ & 8.8 \\
12.5 & $93.07 \pm 1.38$ & $0.44 \pm 0.04$ & 8.5 \\
11.5 & $93.99 \pm 1.44$ & $0.75 \pm 0.06$ & 8.6 \\
10.5 & $94.89 \pm 1.44$ & $1.19 \pm 0.06$ & 9.3 \\
\hline
\end{tabular}

Table 2. Performance comparisons.

\begin{tabular}{|l|l|l|l|l|}
\hline \multicolumn{1}{|c|}{ Method } & \multicolumn{1}{|c|}{ Capacity } & CBP [\%] & Utilization [\%] & $\begin{array}{c}\text { Mean service } \\
\text { time }\end{array}$ \\
\hline WWW & 220 & 0 & $90.04 \pm 1.06$ & $14.94 \pm 1.45$ \\
CAC & 220 & $0.13 \pm 0.06$ & $89.70 \pm 0.80$ & $13.45 \pm 0.98$ \\
\hline WWW & 195 & 0 & $98.66 \pm 0.55$ & $19.67 \pm 2.22$ \\
CAC & 195 & $4.52 \pm 0.82$ & $97.11 \pm 0.35$ & $12.91 \pm 1.18$ \\
\hline WWW-DYN & $212.9 \pm 1.09$ & 0 & $91.23 \pm 1.44$ & $15.63 \pm 1.63$ \\
CAC-DYN & $213.6 \pm 1.92$ & $0.46 \pm 0.04$ & $92.81 \pm 1.86$ & $13.57 \pm 0.84$ \\
\hline
\end{tabular}


The reservation is never less than the actual number of allocated connections $n$. The allocation formula is:

$$
m=\max (n, \quad \neg),
$$

where $n$ is the actual number of allocated connections. It should be noted that if the number of retrials are limited or set to zero, there should be an offset of capacity in the allocation formula (3). The extra capacity needed to reach the target CBP is almost zero for about eight retrials and can be estimated as a linear function of the traffic. According to the results in Figure 4 this function is approximately $45+A \cdot 1.15$, when not allowing any retrials. The actual maximum number of retrials can be estimated based on the application. In case of "surfing" the Web, $R_{\max }$ in formula (2) could for example be set to six, which is about one minute.

In formula (2) there is an assumption that the retrials meets the system, where the occupation distribution will be equal to the mean distribution. Although there is an exponential back off, this is not a correct assumption. However, the difference between the estimated traffic without retrials (first traffic) and the real one, is small. Having a capacity of $190\left(A_{\text {total }}\right.$ is 699.9-768.7), $\hat{A}$ is estimated to 201-206. For a capacity of 200 and 210 ( $A_{\text {total }}$ is 556.3-615.4 and 409.6-468.2), $\hat{A}$ gets 203-205 and 209-210, respectively.

\subsection{Evaluation}

The problem of allowing too many users into the Internet often results in what has been called "World Wide Wait." This effect is a result of the sharing of capacity as well as capacity shortage. When the traffic load reaches a limit where the capacity (of routers or servers) is not enough to handle the total stream of packets, some packets will get lost and TCP connections decrease the transfer rate and will occupy the resources for a longer time. New connections will meet an already congested network, which results in even longer delays.

For fixed rate connections $(8 \mathrm{~kb} / \mathrm{s})$ the time to load one embedded item is 12.44 seconds (i.e. the service time). Table 1 shows the cost function for different settings of an expected service time when using the dynamic allocation (DYN) (3) with CAC and allowing up to eight retrials (traffic $=200$ ). The cost function has a minimum at the theoretical value, which shows that the allocation function works. Table 2 shows a comparison between an ideal fair share allocation without CAC (denoted as WWW), and the use of CAC with up to eight retrials.

We let the method WWW have a maximum of $8 \mathrm{~kb} / \mathrm{s}$ for each connection and if the new calculated capacity is less than the maximal capacity that can be used by the current number of established connections, then the new capacity will be set to the mean value of these capacities. The other methods always enable $8 \mathrm{~kb} / \mathrm{s}$ and capacity is always allocated so that the ongoing connections can be accommodated. The dynamic method uses a reallocation period $\left(T_{\mathrm{u}}\right)$ of one minute. No traffic prediction is used but the estimated traffic is smoothed by taking the mean of the previous estimate and the current measurement. The offered first traffic is fixed at 200. The performance is shown for different amount of allocated capacities. When the available capacity decreases the utilization for WWW increases but so does the service time. Having a dynamic allocation even for WWW the service time will be "acceptable." Although, the utilization for WWW and CAC are similar, the 


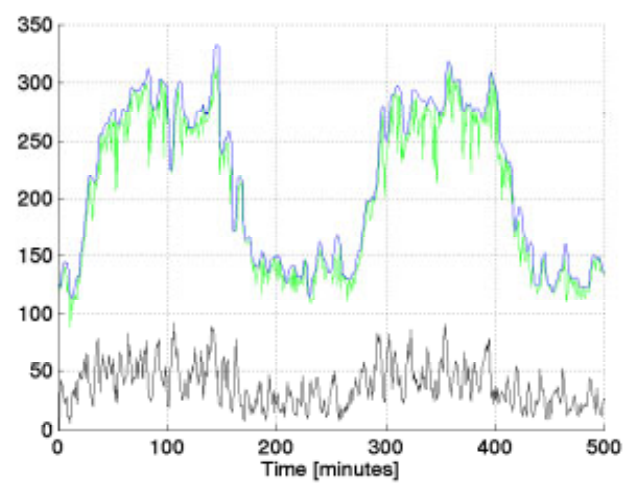

Fig. 7. Dynamic reservation with $T_{\mathrm{u}}=1$ minute.

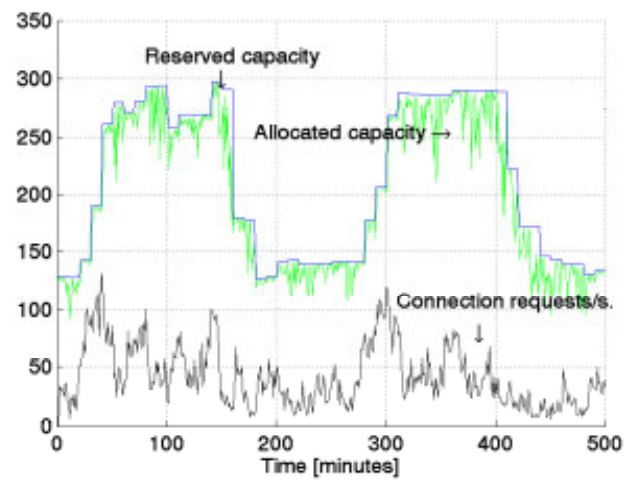

Fig. 8. Dynamic reservation with $T_{\mathrm{u}}=10$ minutes.

distribution of used capacity is different. WWW will have a peak at the maximum capacity, which depends on the fact that no blocking occurs since all connections share the capacity equally. Using retrials, the connections will be delayed and the occupation distribution will increase gradually and reach its peak at the maximum capacity so that the utilization stays high all the time. In the case of having TCP connections, slow start and retransmissions are involved that would have decreased the utilization compared to our results. By using CAC, the overall throughput will increase [8]. Having a control of the transfer rates as well as having proper queuing mechanisms in the switches, the minimum transfer rate is guaranteed.

Evaluations of the performance of CAC-DYN is given in Figures 7 and 8. In this case, the traffic intensity has been varied linearly up and down between steady states that last 100 minutes. $T_{u}$ has been set to one minute and to ten minutes. The bottom curve is the total arrival rate of connection requests as indicated in Figure 8. The resulting blocking probability for $T_{\mathrm{u}}=1$ minute is $0.72 \% \pm 0.05 \%$ with a mean allocated capacity of $214.8 \pm 2.3$. For $T_{\mathrm{u}}=10$ minutes, the blocking is $2.71 \% \pm 0.22 \%$ and a mean allocated capacity of $211.8 \pm 2.0$. 
The evaluation shows that the allocation function works and that sufficient capacity is reserved. By changing the reservation dynamically, the utilization of the capacity does not suffer from the capacity reservation.

\section{Aggregated Traffic}

\subsection{Allocation Function}

In this case we assume that users have limited transfer rates (e.g. by the modem speed) and that LSPs (or similar) with reserved capacity are used for these users. Having different LSPs for different classes of traffic, we can in this case group users, having a certain modem speed, into one class. Our model has similarities to the model shown in Figure 5, except that the LAN is replaced with an Internet subnetwork and the ER with an "aggregation router" [7]. The ER could also correspond to a "bandwidth broker" as in a DiffServ environment. This router, which allocates capacity on LSPs, counts the total packet rate for each LSP. Dividing this bitrate with the user transfer rate for this LSP gives an estimate of the total number of simultaneous Web page fetches. The occupation distribution is modelled as Poissonian and no retrials are permitted here. The needed capacity is calculated with a formula given in $[9,10]$. The allocation can be seen as a confidence interval:

$$
m=\bar{n}+K \sqrt{\bar{n}},
$$

where $\bar{n}$ is the number of simultaneous Web page fetches estimated by the mean of several samples. The transmission speed is not needed in (4) as long as all the users use the same speed (for the LSP in question). It is important to note that (4) can be used in the previous scenario if there is only one connection established per Web page fetch, as proposed for HTML version 1.1 or if the traffic is IP Telephony.

\subsection{Management}

There are basically two methods for reserving capacity. First, if no cost is related to the capacity, all of it should be allocated or reserved (except for some capacity left for best effort services). This can be done by trying to mini-mize the total CBP. When doing this one should take into account the selected $T_{\mathrm{u}}$ and the number of LSPs that share the total capacity on an average link. The available capacity will also affect the result. The setting of $K$ should in this case be done adaptively to take into account the multiplexing effect between LSPs when reallocating frequently. Secondly, if there is some cost related to the reservation of capacity, the cost function has to be optimized, (i.e. just enough capacity is to be used). The setting of $K$ can be made to depend on the cost of allocating the capacity. Another strategy is to have a central network management centre that supports a global control. There are many proposals in the literature for this and for large networks the number of control messages is 


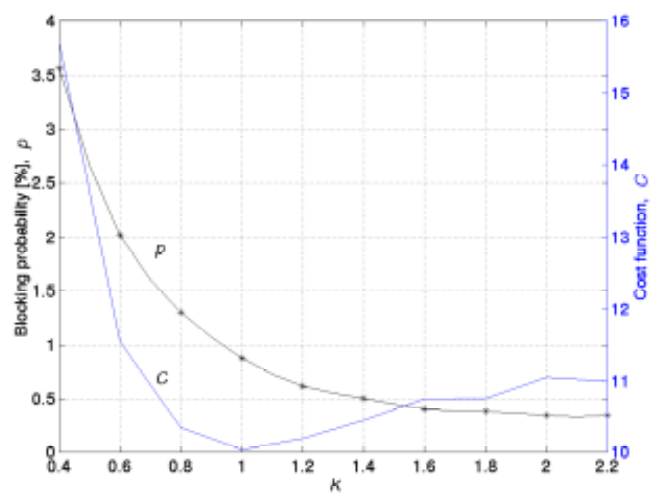

Fig. 9. Blocking probability and cost function for different $K$ s.

minimized. There are, however, situations when a central management is not logical, e.g. in diverse Internet scenarios.

\subsection{Evaluation}

Figure 9 shows the CBP (p) and the value of the cost function when there are four LSPs for $56 \mathrm{~kb} / \mathrm{s}-$ modem users. $T_{\mathrm{u}}$ is set to one minute. The mean transfer time of one object is 1.78 seconds. In the sequel, we denote with $\lambda$ the number of connection arrivals per second. The capacity needed for the LSP in the case of $\lambda=10$ (offered traffic=178) is about $11 \mathrm{Mb} / \mathrm{s}$. The link capacity is set to 4.198 (where $E_{198}$ $(178)=0.01)$.

As seen in the figure the CBP is lower for higher values of $K$. In the case when all the capacity should be utilized, there is a value of $K$ that minimizes the total CBP, which is a little larger than 2.2 in this case. If just enough capacity is to be allocated, then the $K$ is set to 1.0 .

With our allocation function, the CBP decreases for higher traffic. When the traffic intensity increases to $\lambda=20$ the blocking decreases from $0.88 \% \pm 0.02 \%$ to $0.64 \% \pm 0.01 \%$. For $\lambda=30$ the blocking is $0.57 \% \pm 0.02 \%$. These values are very similar to the target blocking probabilities. It is possible to adjust the allocation function to equalize the blocking for different traffics. Blocking probabilities that maximize the total amount of handled connections have greater difference. Setting $K$ to a fixed value can be seen as a trade-off between these extremes of blocking probabilities and further indicate the usefulness of the target blocking probabilities.

\section{Conclusion}

We have described methods to guarantee a certain level of service for Internet traffic by reserving capacity along fixed logical paths. A trade-off between utilization and $\mathrm{CBP}$ is made to enable a method for capacity dimensioning for Internet traffic. If 
consideration is taken to limit the mean service time and increase the throughput, there is an advantage of having CAC. This has also been proposed in [11]. The cost of this is a small CBP. If near real-time services are to be guaranteed some QoS, especially for mobile users, they probably will prefer not to be connected if the service would be less than acceptable. Two allocation functions have been proposed. Each one is tailored for a certain situation of capacity management. In one situation, the network provider manages the capacity. In the other case, capacity is paid for according to how much of it that is reserved.

\section{Future Work}

The problem is to find a cost for reallocations and a cost for not doing them frequent enough. Performance of TCP connections in a realistic network model together with capacity realloc-ations will show how users perceive the performance, which also will be a parameter for the calculation of this cost.

\section{References}

1. P. Pruthi, A. K. Jena, and A. Popescu, "HTTP Interactions with TCP," 11 'th ITC Specialist Seminar, Japan, pp. 195-204, 1998.

2. M. F. Arlitt and C. L. Williamson, "Web Server Workload Character-ization: The Search for Invariants," IEEE/ACM Trans. on Netw., Vol. 5, No. 5, pp. 631-645, 1997.

3. P. Barford and M. E. Crovella, "Generating Representative Web Workloads for Network and Server Performance Evaluation", Proc. on Performance'98 / SIGMETRICS'98, pp. 151160, 1998.

4. A. K. Jena, "Modelling and Evaluation of Internet Applications", Doctoral thesis, ISSN 1101-3931, Lund 2000.

5. R. Guérin and H. Ahmadi, "Equivalent Capacity and Its Application to Bandwidth Allocation in High-Speed Networks," IEEE J. Sel. Areas in Commun., Vol. 9, No. 7, pp. 968-981, Sept. 1991.

6. N. Bhatti and R. Friedrich, "Web Server Support for Tiered Services," IEEE Network, Vol. 13, No. 5, pp. 64-71, 1999.

7. P. Goyal, et al., "Integration of Call Signalling and Resource Management for IP Telephony," IEEE Network, Vol. 13, No. 3, pp. 24-32, 1999.

8. A. Kumar, et al., "Nonintrusive TCP Connection Admission Control for Bandwidth Management of an Internet Access Link," IEEE Commun. Magazine, pp. 160-167, May 2000.

9. J. Virtamo and S. Aalto, 'Remarks on the effectiveness of dynamic VP bandwidth management," COST 257 TD(97)15, Jan. 1997.

10.S. O. Larsson and Å. Arvidsson, "Performance Evaluation of a Local Approach for VPC Capacity Management," IEICE Trans. on Com., Vol. E81-B, No. 5, pp. 870-876, 1998.

11.J. W. Roberts and L. Massoulié, "Bandwidth sharing and admission control for elastic traffic," 11 'th ITC Specialist Seminar, Japan, pp. 263-271, 1998.

12.X. Xiao, et al., "Traffic Engineering with MPLS in the Internet," IEEE Network, Vol. 14, No. 2, pp. 28-33, 2000.

13.S. Shenker, R. Braden, and D. Clark, “Integrated Service in the Internet Architecture: An Overview," IETF RFC1633, July 1994.

14.R. Braden, et al., "Resource ReServation Protocol (RSVP) - Version 1 Functional Specification," IETF RFC2205, Sept. 1997. 
15.J. Wroclawski, "Specification of the Controlled-Load Network Element Service," IETF RFC2211, Sept. 1997.

16.S. Shenker, R. Braden, and D. Clark, “An Architecture for Differentiated Services," IETF RFC2475, Dec. 1998. 